\title{
Factors Associated with Quality of Life in Huntington's Disease
}

Najwa Hanim Md Rosli

Department of Psychiatry, International Islamic University Malaysia, Kuantan, Pahang, Malaysia

Introduction: Huntington's disease (HD) is an inherited progressive neurodegenerative disorder which affects motor function, cognition and the psychological state of HD patients. Its triad of unique symptoms compared to other neurodegenerative disorders might give rise to a higher level of burden for their carers. The aim of this study was to explore factors associated with the quality of life in patients with HD. Materials and Method: Eighty-one HD patients and their carers participated in this study. Cognitive impairment was assessed with the Neuropsychiatry Unit Cognitive Assessment Tool (NUCOG), motor disturbances was scored using the Unified Huntington's Disease Rating Scale (UHDRS) motor scale, psychological status of both patient and carer were assessed using the Depression Anxiety Stress scale (DASS21), behavior of the patient was assessed using Revised Cambridge Behavioural Inventory (CBI-r) and patient's quality of life was assessed with WHOQOL-BREF questionnaire. Results: Patients' psychological conditions, motor disturbances and behavior of the patient are correlated with low QOL in HD patient. Conclusion: Other than regular motor symptoms, psychological and behaviour of HD assessment should be assessed regularly to ensure the patients having good quality of life. 